\title{
RETRACTED ARTICLE: Acute and chronic toxicity of the pharmaceutical levonorgestrel to the freshwater crustacean Daphnia magna
}

\author{
A. L. Oropesa - L. Guimarães · A. Martins • \\ F. Soler · L. Guilhermino
}

Accepted: 8 November 2011/Published online: 17 November 2011

(C) Springer Science+Business Media, LLC 2012

This article has been retracted due to copyright issues that cannot be resolved.

\footnotetext{
A. L. Oropesa $(\varangle) \cdot$ F. Soler

Toxicology Area, Veterinary Faculty, University of

Extremadura, Avda. de la Universidad s/n, P.O. Box 643,

10003 Cáceres, Spain

e-mail: aoropesa@unex.es

F. Soler

e-mail: solertox@unex.es

L. Guimarães · A. Martins · L. Guilhermino

Laboratory of Ecotoxicology and Ecology, CIIMAR-

Interdisciplinary Center for Marine and Environmental,

University of Porto, Rua dos Bragas, 289,

4050-123 Porto, Portugal

e-mail: lguimaraes@ciimar.up.pt
}

A. Martins

e-mail: amartins@ciimar.up.pt

\footnotetext{
A. Martins - L. Guilhermino

Laboratory of Ecotoxicology, Department of Populations

Studies, ICBAS-Instituto de Ciências Biomédicas de Abel

Salazar, University of Porto, Lg. Prof. Abel Salazar, 2,

4099-003 Porto, Portugal

e-mail: lguilher@icbas.up.pt
} 